\title{
Comparison of Neutrophil to Lymphocyte Ratio in Obstructive Sleep Apnea Syndrome Phenotypes
}

\author{
(D) Berk Gürpınar ${ }^{1}$, (D) Cengiz Özdemir², (1) Belgin Tutar ${ }^{1}$ \\ 1 University of Health Sciences Turkey, Prof. Dr. Cemil Taşşığlu City Hospital, Clinic of Otorhinolaryngology, Istanbul, Turkey \\ 2 Yedikule Chest Diseases Hospital, Clinic of Sleep, Istanbul, Turkey
}

\section{Abstract}

Objective: Our aim in this study was to compare the neutrophil to lymphocyte ratio (NLR) in rapid eye movement (REM) related obstructive sleep apnea (RRO) syndrome (OSAS) and position related OSAS (SRO) to habitual snorers (HSN) and try to find out the phenotype that increases the physical stress more.

Methods: The patient charts and polysomnograms of all subjects referred to Yedikule medical center for chest diseases sleep department between June 2019 and January 2020 were reviewed and classified as RRO, SRO and HSN. Fasting blood tests were performed in the morning following polysomnography. The phenotypes of OSAS were compared to each other by means of NLR.

Results: A total number of 147 cases were reviewed. Forty eight cases were SRO, 49 cases were RRO and 50 were simple snorer. The NLR in RRO was statistically significant from those of SRO and HSN subgroups $(p=0.001)$, whereas NLR in groups SRO and HSN were identical $(p=0.650)$. Apnea Hypopnea index and $\mathrm{O}_{2}$ nadir in all groups were statistically significant from each other.

Conclusion: Our data suggests that the REM related OSAS increases NLR as a predictor of inflammation, but NLR stays constant in Position related OSAS and simple snorers.

Keywords: Obstructive sleep apnea syndrome, REM sleep, supine position, snoring

\section{INTRODUCTION}

Obstructive Sleep Apnea syndrome (OSAS) is a sleep breathing disorder that has potential cardiovascular and/or neurologic complications. The most prominent feature of OSAS is apnea or hypopnea that decreases or blocks the upper airway passage. OSAS is a complex disease; recent studies suggested certain phenotypes for OSAS; those phenotypes result from the combination of the genotype of the susceptible individual and the environmental effects. Up-to-date, the most common phenotypes of OSAS are named to be rapid eye movement (REM)-related OSAS (RRO) as a result of hyporesponsive genioglossal muscle activity that results in hypopharyngeal blockage of the airway by the tongue base during the REM period and secondly supine related OSAS (SRO) phenotype that restricts patients sleeping in the supine position (1).

OSAS may generally be attributed as an inflammatory disorder because of its physical load of distress due to hypoxia/ hypoxemia and increased effort to breathe (2). Neutrophil to lymphocyte ratio (NLR) is a frequently used marker for subclinical inflammation. Basically, the blood drawn peripherally is used and the number of neutrophils is divided by the number of Iymphocytes. Under physiologic stress, by the catalysing effect of the endogenous cortisol and catecholamines, the neutrophils increase and lymphocytes decrease. Normally, NLR in healthy adults are between 1 and 3. However, a mild inflammatory response increases the NLR to 6-9, whereas critically ill patients 
will often have an NLR of 9 or higher, occasionally reaching values close to $100(3,4)$.

NLR has been studied in vast areas of medicine including sleep medicine. It has been shown that OSAS induces physical stress that raises the inflammatory markers such as fibrinogen, C-reactive protein (CRP) and NLR (5). However, to the best of our knowledge the phenotypes of OSAS are not studied nor compared to each other.

Our aim in this study is to compare the NLR in RRO and SRO to habitual snorers (HSN) and try to find out the phenotype that increases the physical stress more.

\section{METHODS}

This was a retrospective study performed in a tertiary care referral center. The ethical approval was granted from the Ethical Committee of Okmeydanı Training and Research Hospital on 21.01.2020 (48670771-514.10). Informed consents were taken from each and every case. The patient charts and polysomnograms of all subjects referred to Yedikule medical center for chest diseases sleep department between June 2019 and January 2020 were reviewed and classified as RRO, SRO, HSN or unclassified OSAS. Unclassified OSAS was referred to as OSAS that has Apnea Hypopnea index (AHI) greater than 5 events/h but not has any specific feature related to REM stage of the sleep nor worsens due to supine position. For being beyond the scope of this study, unclassified OSAS cases were excluded from the study.

Adequate sample size formulation for the study was calculated as follows;

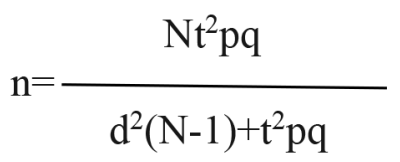

where;

$\mathrm{N}$ : Sample size in the universe (Frequency of patients been treated in our clinic within one month)

$\mathrm{n}$ : The desired sample size

$\mathrm{p}$ : The frequency of occurrence (OSAS incidence was accepted as $7 \%$ )

$\mathrm{q}$ : The frequency of non-occurrence

t: t value

d: Absolute precision or accuracy, normally set at 0.05

$$
\mathrm{n}=\frac{(8000)(1.96) 2(0.07)(0.93)}{(0.05) 2(8000-1)+(1.96) 2(0.07)(0.93)}=98
$$

This equation showed that 98 cases or more were adequate for sampling, and our cases were above this value.

Full-night attended polysomnograms including sixteen channel electroencephalography, two channel electrooculography, submental and left/right anterior tibial electromyography (EMG), right/left biceps surface EMG, body position probe, electrocardiography, oro-nasal 4 thermal sensor, nasal pressure sensor, breath sound recording, thoracal and abdominal sensors, pulse oxymeter and synchronous video recordings were recorded. Wake-sleep patterns and sleep related abnormal breathing events were scored with respect to the criteria of American Academy of Sleep Medicine 2017 (AASM). All polysomnograms were evaluated due to AASM-International Classification of Sleep Disorders international sleep diseases 2014 criteria. PSG recordings of all subjects were scored by a sleep expert who was blinded to blood test results of the cases.

As a routine, fasting blood tests were performed in the morning following polysomnography. Detailed parameters included complete blood count, blood glucose, urea, liver function tests, fibrinogen, erythrocyte sedimentation rate, CRP, ferritin and iron levels.

Exclusion criteria included as follow: pediatric age group, subjects other than sleep disordered breathing problems (i.e. insomnia, parasomnia, circadian rhythm disorders, etc.), previous upper airway surgery, any kind of central sleep apnea, upper airway neoplasm and acute rhinitis resulting in nasal obstruction at the time of the study. Obstructive apnea was defined as the total cessation of airflow for $\geq 10 \mathrm{~s}$ with continued respiratory effort. Obstructive hypopnea was defined as an event lasting $\geq 10 \mathrm{~s}$ that was associated with both a decrease in airflow of $50 \%$ and an EEG arousal or oxygen desaturation $\geq 4 \%$. AHI was defined as the number of apneic and hypopneic events per hour of sleep.

The predominance of disordered breathing during REM sleep was accepted as: (a) an AHI of nonREM sleep stage fewer than 5 events/h and (b) an AHI of REM sleep stage of at least 5 events/h with at least 30 minutes of REM sleep. If the amount of REM sleep was less than 30 minutes, estimates of AHI REM were considered imprecise and the cases were excluded (6). The predominance of disordered breathing during supine position sleep was accepted as: (a) Overall AHI is greater than 5 events/h, (b) The supine AHI is greater than two times the non-supine AHI and, (c) Non-supine 
$\mathrm{AHI}$ is less than 5 events/h (7). Habitual (simple) Snorers were defined as snorers with an $\mathrm{AHI}<5$ events/h.

\section{Statistical Analysis}

Statistical analyses were performed by SPSS 22.0 (IBM, Turkey). A power analysis revealed our sample size to be adequate for the study. A Shapiro-Wilks test was used to control the normal distribution of the variables. Continuous variables were analyzed by one-way variance analysis test and inter-group comparisons were performed by Post-Hoc Bonferroni tests. Continuous variables were expressed as mean, standard deviation, minimum, maximum and categorical variables as frequency and percentage. A p value of $<0.05$ was accepted statistically significant.

\section{RESULTS}

A total number of 147 cases were reviewed. Forty eight cases were SRO (32.65\%; 36 male, 12 female), 49 cases were RRO (33.33\%; 28 male, 21 female) and 50 were simple snorer (34.01\%; 27 male, 23 female). Descriptive variables are presented in Table 1.

The NLR in RRO was statistically significant from those of SRO and HSN subgroups ( $p=0.001)$; RRO had the highest value as of 2.76, SRO had 1.53 and HSN had the lowest value as 1.37. The Post-Hoc values between RRO and HSN was significant $(p=0.001)$, however NLR difference in HSN and SRO $(p>0.005)$ or SRO and RRO ( $p>0.005)$ were not significant. $\mathrm{AHI}$ and $\mathrm{O}_{2}$ nadir in all groups were statistically significant from each other as SRO 16.6; RRO 10.1 and HSN 2.3. Also the Post-Hoc values of AHI was statistically significant between RRO and HSN being worse for RRO ( $p=0.001)$, HSN and SRO, being worse for SRO $(p=0.001)$, and SRO and RRO, being worse for SRO $(p=0.005)$ (Table 2 , Figure 1).

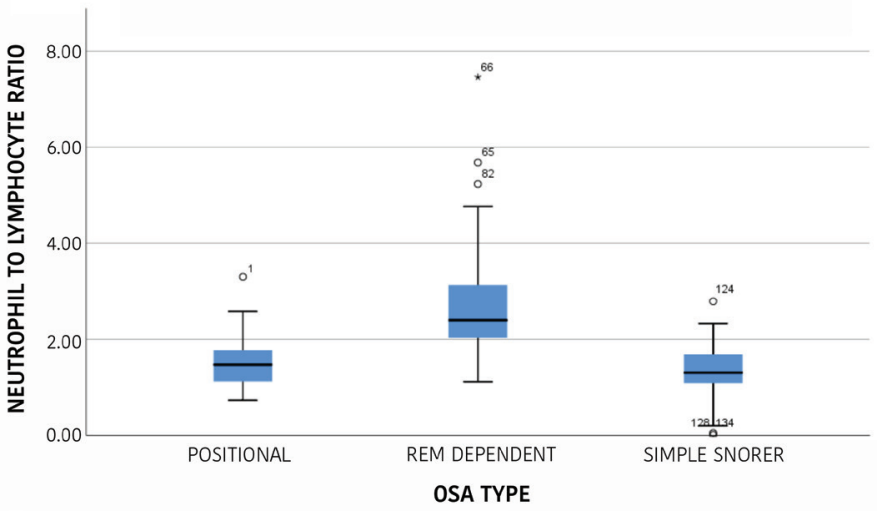

Figure 1. Simple Boxplot of neutrophil to lymphocyte ratio by OSA type OSA: Obstructive Sleep Apnea

\begin{tabular}{|c|c|c|c|c|c|c|}
\hline \multicolumn{2}{|c|}{ One-way ANOVA } & $\mathbf{n}$ & Mean & Standard deviation & Minimum & Maximum \\
\hline \multirow[t]{3}{*}{ Age } & SRO & 48 & 45.93 & 10.18 & 25.00 & 66.00 \\
\hline & RRO & 49 & 48.71 & 10.37 & 25.00 & 74.00 \\
\hline & HSN & 50 & 45.32 & 10.79 & 25.00 & 74.00 \\
\hline \multirow[t]{3}{*}{ BMI } & SRO & 48 & 30.79 & 3.93 & 25.00 & 41.00 \\
\hline & RRO & 49 & 31.95 & 7.03 & 22.00 & 50.00 \\
\hline & HSN & 50 & 30.20 & 4.79 & 21.00 & 41.00 \\
\hline \multirow[t]{3}{*}{ NLR } & SRO & 48 & 1.53 & 0.55 & 0.73 & 3.30 \\
\hline & RRO & 49 & 2.76 & 1.25 & 1.12 & 7.46 \\
\hline & HSN & 50 & 1.37 & 0.54 & 0.01 & 2.79 \\
\hline \multirow[t]{3}{*}{ TST } & SRO & 48 & 411.77 & 45.12 & 275.00 & 530.50 \\
\hline & RRO & 49 & 406.02 & 57.49 & 230.50 & 481.00 \\
\hline & HSN & 50 & 386.34 & 61.25 & 213.00 & 528.00 \\
\hline \multirow[t]{3}{*}{$\mathrm{AHI}$} & SRO & 48 & 16.61 & 9.58 & 5.70 & 39.50 \\
\hline & RRO & 49 & 10.10 & 4.01 & 5.40 & 21.70 \\
\hline & HSN & 50 & 2.30 & 1.33 & 0.00 & 4.90 \\
\hline \multirow[t]{3}{*}{ Oxygen nadir } & SRO & 48 & 88.33 & 4.83 & 77.00 & 97.00 \\
\hline & RRO & 49 & 84.75 & 7.49 & 56.00 & 96.00 \\
\hline & HSN & 50 & 92.58 & 3.16 & 85.00 & 97.00 \\
\hline
\end{tabular}




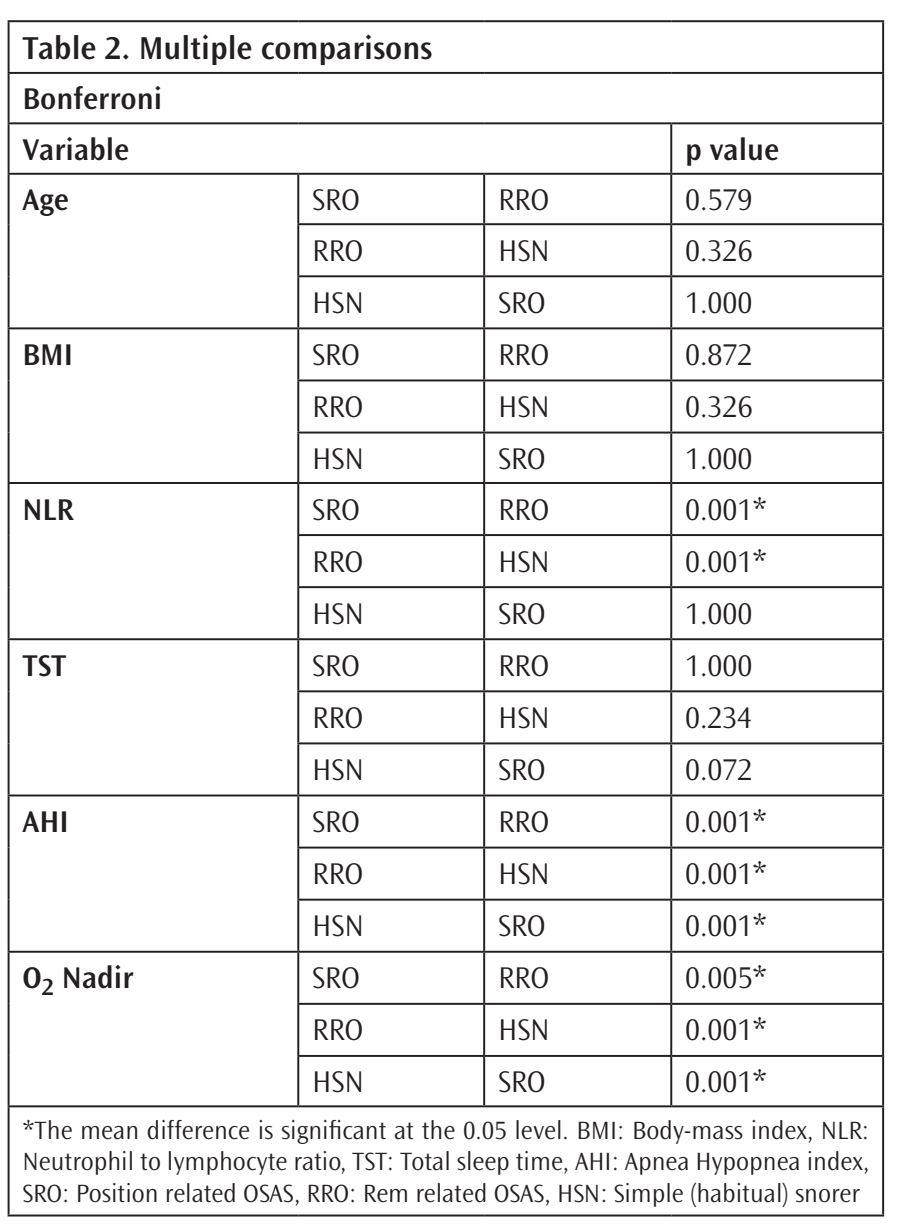

\section{DISCUSSION}

OSAS is a chronic disease that has repetitive episodes of upper airway collapse and then relievement of the collapse by gasping of the susceptible subject, which in turn causes low-level systemic inflammation in a certain time period. It has been linked to a diverse variety of comorbidities such as cardiovascular diseases, pulmonary diseases, endocrine dysfunctions, and neuropsychiatric problems. The disease, furthermore, also causes hypoxia, asphyxia, respiratory acidosis and hypercapnia, which are due to the reoccurring sleep disruptions and leads to the development of local and systemic inflammation. Chronic inflammation is thought to be the underlying pathogenesis, although the exact physiopathology to those health problems remains unclear. However, the inflammation might be the result or the cause of these pathologies; more commonly a bidirectional relationship is frequently questioned (8).

It has been demonstrated in an animal model that apnea and hypoxemia trigger systemic inflammation by inducing changes in the leukocyte function (9). Under physiologic stress, the number of neutrophils increases, while the number of lymphocytes decreases. The NLR combines both of these changes.

Previous reports demonstrated that patients with OSAS have higher levels of inflammatory markers such as CRP, neutrophil counts, TNF- $\alpha$, pro-inflammatory cytokines, interleukin 6 , serum amyloid A, and leukocyte adhesion molecules $(10,11)$.

Obstructive sleep apnea repetitively fragments the sleep. This fragmentation and inflammatory response increases the sympathetic neural activity. Elevation of the plasma noradrenaline activates the sympathetic nervous system and elevates the serum cortisol. Once the plasma cortisol level increases, a decrease in the relative concentration of lymphocytes occurs and this low level of lymphocytes worsens the clinical outcome. Neutrophils are the frontiers of the defensive cells of the immune system but an uncontrolled release of toxic substances such as inflammatory cytokines or tissue-damaging enzymes may potentiate the damage to surrounding tissues and increase the inflammatory responses. An increase in the neutrophil count of OSAS patients were shown but the reason why neutrophils are increased in the airways of OSAS patients remains unclear. No matter how different mechanisms could be involved, intermittent hypoxia is thought to be the major factor for this phenomenon (12).

In this study, particular importance is attributed to REM sleep. During the REM sleep; the electrical activity of the brain is similar to being awake when measured by oxygen and glucose metabolism being $11-40 \%$ lower in non-REM sleep. Areas of the brain that are active during REM sleep are the opposite of the ones activated during non-REM sleep. Especially, the amygdala is active during REM sleep which is involved in autonomic responses associated with fear and hormonal secretions. Scientific studies of the amygdala have led to the discovery of the location of neurons in the amygdala that are responsible for fear conditioning. Body temperature is not well-regulated which can cause to become uncomfortable with more extreme temperatures. The inhibition of motor neurons causes a complete paralysis of the body and REM sleep is the part of sleep where dreams occur; the brain will often deploy "safety measures" to ensure it isn't disrupted. For example, the sound of an alarm clock or phone may be incorporated into the dream and transformed into something else. All those features associated with REM sleep causes increased heart rate and catecholamines and shallow breath. Additional inhibition of the skeletal muscles increases the apnea/hypopnea events and hypoxemia deepens (13). All abovementioned responses load important stress level to the affected body and the stress levels 
gradually increase throughout the evolution of the disease. Those mechanisms were in concordance with our study; the NLR ratio of REM was significantly higher than the other two groups. We accepted RRO to have a REM period equal or greater than 30 minute. However, the time variable was not a constant valid rule for SRO. This might be an important clue to explain why RRO NLR was higher than HSN but SRO was not statistically significant than HSN. If the standardization of the time variable spent in both REM sleep and supine position sleep should be set as 30 minutes, maybe the scores would change and NLR of both OSAS phenotypes might be the same. We believe further researches in the area illuminating this point could be of importance.

\section{CONCLUSION}

The literature reviews support the NLR increase in general OSAS, but the phenotypes were not studied before. Our data suggests that the REM related OSAS increases NLR as a predictor of inflammation, but NLR stays constant in Position related OSAS and simple snorers.

\section{Ethics}

Ethics Committee Approval: Ethical Committee of Okmeydanı Training and Research Hospital on 21.01.2020 (48670771514.10).

Informed Consent: Informed consents were taken from each and every case.

Peer-review: Externally and internally peer-reviewed.

\section{Authorship Contributions}

Concept: B.G., C.Ö., Design: B.G., C.Ö., Data Collection or Processing: B.G., B.T., C.Ö., Analysis or Interpretation: B.G., B.T., Literature Search: B.G., B.T., Writing: B.G.

Conflict of Interest: No conflict of interest was declared by the authors.

Financial Disclosure: The authors declared that this study received no financial support.

\section{REFERENCES}

1. Subramani Y, Singh M, Wong J, Kushida CA, Malhotra A, Chung F. Understanding Phenotypes of Obstructive Sleep Apnea: Applications in Anesthesia, Surgery, and Perioperative Medicine. Anesth Analg 2017;124:179-91.

2. Unnikrishnan D, Jun J, Polotsky V. Inflammation in Sleep Apnea: An Update. Rev Endocr Metab Disord 2015;16:25-34.

3. Zahorec R. Ratio of neutrophil to lymphocyte counts--rapid and simple parameter of systemic inflammation and stress in critically ill. Bratisl Lek Listy 2001;102:5-14.

4. Karakonstantis S, Kalemaki D, Tzagkarakis E, Lydakis C. Pitfalls in studies of eosinopenia and neutrophil-to-lymphocyte count ratio. Infect Dis (Lond) 2018;50:163-74

5. Kheirandish-Gozal L, Gozal D. Obstructive Sleep Apnea and Inflammation: Proof of Concept Based on Two Illustrative Cytokines. Int J Mol Sci 2019:20

6. Mokhlesi B, Punjabi NM. "REM-related" Obstructive Sleep Apnea: An Epiphenomenon or a Clinically Important Entity? SLEEP 2012;35:5-7.

7. Dieltjens M, Braem MJ, Van de Heyning PH, Wouters K, Vanderveken MO. Prevalence and Clinical Significance of Supine-Dependent Obstructive Sleep Apnea in Patients Using Oral Appliance Therapy. J Clin Sleep Med 2014;10:959-64.

8. Ataç E, Yıldırım G, Kumral TL, Berkiten G, Saltürk Z, Uyar Y. Obstructive Sleep Apnea Syndrome and Obesity. Eur Arc Med Res 2014;30(Suppl 1):19-23.

9. Levy P, Pepin JL, Arnaud C, Tamisier R, Borel JC, Dematteis M, et al Intermittent hypoxia and sleep-disordered breathing: current concepts and perspectives. Eur Respir J 2008;32:1082-95.

10. Bravo Mde L, Serpero LD, Barceló A, Barbé F, Agustí A, Gozal D. Inflammatory proteins in patients with obstructive sleep apnea with and without daytime sleepiness. Sleep Breath 2007;11:177-85.

11. Dyugovskaya L, Lavie P, Lavie L. Inycreased adhesion molecules expression and production of reactive oxygen species in leukocytes of sleep apnea patients. Am J Respir Crit Care Med 2002;165:934-9.

12. Korkmaz M, Korkmaz H, Küçüker F, Ayyıldız SN, Çankaya S. Evaluation of the Association of Sleep Apnea-Related Systemic Inflammation with CRP ESR, and Neutrophil-to-Lymphocyte Ratio. Med Sci Monit 2015;21:47781

13. Fraigne JJ, Torontali ZA, Snow MB, Peever JH. REM Sleep at its CoreCircuits, Neurotransmitters, and Pathophysiology. Front Neurol 2015;29;123. 